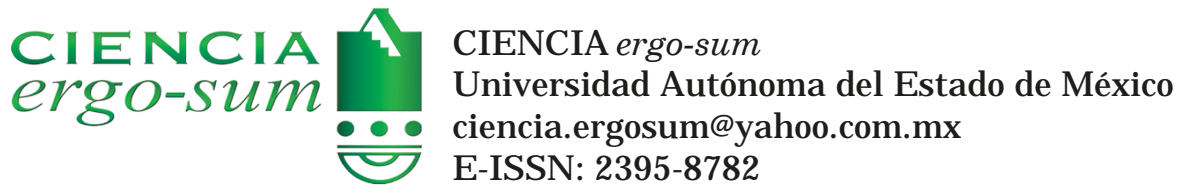

\title{
Autorreporte de enfermedades y patrón alimentario de adultos $\geq 50$ años sedentarios vs. físicamente activos
}

\author{
Ramos-J iménez, Arnulfo; Hernández-Torres, Rosa Patricia; Wall-Medrano, Abraham; J uárez-Oropeza, \\ Marco Antonio \\ Autorreporte de enfermedades y patrón al imentario de adultos $\geq 50$ años sedentarios vs. físicamente activos \\ CIENCIA ergo-sum, vol. 26, núm. 3, noviembre 2019-febrero 2020| e63 \\ Universidad Autónoma del Estado de México, México
}

Esta obra está bajo una Licencia Creative Commons Atribución-NoComercial-SinDerivar 4.0 Internacional.

Ramos-J iménez, A., Hernández-Torres, R. P., Wall-Medrano, A. y J uárez-Oropeza, M. A. (2019). Autorreporte de enfermedades y patrón alimentario de adultos $\geq 50$ años sedentarios vs. físicamente activos. $\mathrm{CIE} \mathrm{NCIA} \mathrm{ergo-}$ sum, 26(3). https://doi.org/10.30878/ces.v26n3a6 


\title{
Autorreporte de enfermedades y patrón alimentario de adultos $\geq$ 50 años sedentarios vs. físicamente activos
}

Disease self-report and food patterns of sedentary vs. physically active adults $\geq 50$ years

\author{
Arnulfo Ramos-Jiménez \\ Universidad Autónoma de Ciudad Juárez, México \\ aramos@uacj.mx \\ Rosa Patricia Hernández-Torres \\ Universidad Autónoma de Chihuahua, México \\ rphernant@yahoo.com \\ Abraham Wall-Medrano \\ Universidad Autónoma de Ciudad Juárez, México \\ awall@uacj.mx \\ Marco Antonio Juárez-Oropeza \\ Universidad Nacional Autónoma de México, México \\ majo_ya@yahoo.com.mx
}

\section{RESUMEN}

Se investiga la relación entre el nivel de actividad física (NAF) con el patrón alimentario y autorreporte de enfermedades en adultos $\geq$ 50 años del norte de México. Se les registraron presión sanguínea, dimensiones antropométricas, NAF (MET-min/semana), hábitos alimentarios y autorreporte de enfermedades a 200 participantes (50-80 años; 52\% mujeres). Los resultados muestran que $25 \%$ es sedentario, $42 \%$ es activo moderadamente y $34 \%$ es muy activo. Los sedentarios presentaron mayor peso $(+9 \mathrm{~kg})$, circunferencia de cintura $(+8.1 \mathrm{~cm})$, número $(+2)$ y variedad $(10-23)$ de enfermedades que la de grupos activos $(p<0.05)$; el consumo de alimentos densamente energéticos fue menor en personas muy activas $(f \geq 0.27)$. Las conclusiones muestran que un NAF $\leq 1137 \mathrm{MET}$-min/ semana (bajo-moderado) aumenta el riesgo de enfermedades en personas adultas $(r=-0.15$ a -0.33$)$.

Palabras Clave: adulto mayor, ejercicio físico, hábitos alimentarios.

\begin{abstract}
Objective: To investigate the relationship between the level of physical activity (LPA) with the eating pattern and self-reported diseases in adults $\geq 50$ years in northern Mexico. Methodology: To 200 participants ( $50-80$ years, 52\% women) blood pressure, anthropometric dimensions, LPA (MET-min/week), eating habits and self-reported diseases were registered. Results: $25 \%$, 42\%, and 34\% were sedentary, moderately and very active, respectively. Sedentary subjects presented higher weight $(+9 \mathrm{~kg})$, waist circumference $(+8.1 \mathrm{~cm})$, number $(+2)$, and variety $(10-23)$ of self-reported diseases than active groups $(p<0.05)$. The consumption of densely energetic foods was lower in very active people $(f \geq 0.27)$. Conclusion: A LPA of 1137 MET-min/week (low-moderate) increases the risk of diseases in adults $(r=-0.15$ to -0.33$)$.
\end{abstract}

KEYWORDS: older adult, physical exercise, eating habits.

\section{INTRODUCCIÓN}

La pluripatología durante la transición adulto-adulto mayor no sólo es resultado del deterioro orgánico asociado a la edad, sino también a otros factores; en particular, la transición alimentaria/nutricional (Tourlouki et al., 2009) y el sedentarismo observables en el adulto mayor (Sun et al., 2013) se encuentran estrechamente relacionados con 
el número de enfermedades crónicas no transmisibles (ECNT) qué este padece (Sparling et al., 2015). En este tenor, se calcula que en el mundo $70 \%$ de las muertes es debido a enfermedades crónicas no transmisibles (GBD, 2016). En México la proporción de adultos mayores va en aumento: en 2010 se contabilizaron cerca de siete millones de adultos mayores de 65 años (6.2\% de la población) (INEGI, 2013) y la esperanza de vida actual del mexicano es de 75 años, por lo que se incrementa la necesidad de servicios asistenciales (incluyendo los de salud) para este segmento poblacional (González-González et al., 2011). Además, la transición nutricional y estilo de vida del adulto y adulto mayor mexicano inciden en su transición epidemiológica hacia las ECNT demeritando su calidad de vida (Gutierres et al., 2012; Kushner y Choi, 2010; Varo et al., 2003). Al respecto, de acuerdo con la Secretaría de Salud en 2012 existían 1.5 millones de personas $\geq 50$ años con los siguientes padecimientos: obesidad (29\%), diabetes (23\%), hipertensión (23\%) e hipercolesterolemia (19\%), entre otros (Gutierres et al., 2012), y se confirma en la Encuesta Nacional de Salud y Nutrición de Medio Camino 2016 (ENSANUT MC) que estas prevalencias no han descendido (INSP, 2016).

Varios estudios epidemiológicos y metaanálisis han documentado el aumento del sedentarismo con la edad (Owen et al., 2010) y el aumento en las tasas de obesidad (Varo et al., 2003), hiperlipidemias, osteoporosis (Tremblay et al., 2010), síndrome metabólico (Ford et al., 2005), algunos tipos de cáncer y muerte prematura (Haskell et al., 2007).En la ENSANUT MC 2016 (INSP, 2016) se reportó un muy bajo porcentaje de sedentarismo (14.4\%) en la población $\geq 20$ años (INSP, 2016), aunque en las encuestas pasadas la realidad fue contraria (Medina et al., 2013). El Colegio Americano de Medicina del Deporte recomienda realizar al menos 30 minutos de ejercicio moderado (3-6, METs o equivalentes metabólicos) o vigoroso (> 6 METs) cinco días por semana (Haskell et al., 2007), los cuales no se alcanzan en la sociedad moderna (Hamilton et al., 2008).

Respecto a la alimentación, los cambios en la dieta, con y sin ejercicio físico, han probado reducir el sobrepeso, la obesidad, los riesgos a enfermedades cardiovasculares (Smith et al., 2010) y a mejorar el estado de salud general de la población, así como su calidad de vida (Drewnowski y Evans, 2001; Goodpaster et al., 2010). Sin embargo, la implementación de programas nutricionales saludables no ha sido una tarea fácil, en especial entre personas adultas, sobre todo si no se acompañan de políticas para el establecimiento de un ambiente cultural y saludable nutricionalmente (Bimbo et al., 2015; Hoffman, 2010). Al menos en México las políticas nutricionales no han tenido el efecto esperado, ya que no se ha reducido la alta tasa de obesidad de la población (Barquera et al., 2001; INSP, 2016). En relación con los hábitos alimentarios, las personas adultas del norte de México mencionan que comen menos cantidad de frutas y verduras ( $47 \%$ y $35 \%$ respectivamente) y mayor cantidad de legumbres, huevo y carnes procesadas (78\%, 67\% y $87 \%$ respectivamente) en relación con la media nacional (INSP, 2016).

Por lo anterior, el propósito de este artículo es investigar la relación entre el nivel de actividad física (NAF) con el patrón alimentario y autorreporte de enfermedades en adultos $\geq 50$ años del norte de México.

\section{Materiales y Métodos}

\section{1. Participantes}

Bajo un diseño transversal, observacional no probabilístico, se estudiaron 200 voluntarios $\geq 50$ años (50-80 años) de ambos sexos (105 mujeres) de Ciudad Juárez Chihuahua: 50 sedentarios (27 mujeres), 83 moderadamente activos (52 mujeres) y 67 muy activos (26 mujeres). Este trabajo definió como sedentarios a aquellos que no realizan ningún tipo de actividad deportiva o recreativa, como moderadamente activos a quienes realizan entre 600 a $1500 \mathrm{MET} / \mathrm{min} /$ semana de ejercicio y como muy activos más de $1500 \mathrm{MET} / \mathrm{min} / \mathrm{semana}$ de actividad física; lo anterior de acuerdo con los criterios del Cuestionario Internacional de Actividad Física (IPAQ, por sus siglas en inglés) y los lineamientos de actividad física establecidos para adultos mayores (Haskell et al., 2007; Nelson et al., 2007). Los criterios de inclusión fueron tener $\geq 50$ años de edad, no fumar o presentar problemas músculo-esqueléticos u otros padecimientos que les impidiera llevar a cabo actividades físicas, no haber ingerido alcohol al menos en 48 h, café u otros fármacos que 
afectaran el estado de hidratación y firmar la carta de consentimiento informado de acuerdo con los lineamientos de la carta de Helsinki. El protocolo fue aprobado por el Comité de Ética de la Universidad Autónoma de Ciudad Juárez.

\subsection{Procedimientos}

Para el día de la entrevista se solicitó a las personas asistir con al menos $24 \mathrm{~h}$ posteriores al ejercicio físico extenuante y con al menos $2 \mathrm{~h}$ de ayuno. El mismo día se aplicó el IPAQ versión corta (Craig et al., 2003), un cuestionario sobre hábitos alimentarios (Pino et al., 2011), adaptado para el norte de México (no publicado), y un cuestionario sobre el estado de salud general del deportista (American College of Sports Medicine, 2013). Además, se midió la presión sanguínea (baumanómetro aneroide Reister Precisan, USA) después de 10 min de estar sentado y la antropometría (peso, estatura y circunferencia de cintura) de acuerdo con los lineamientos de la Sociedad Internacional para el Avance de la Kinantropometría (ISAK, por sus siglas en inglés) (Norton y Olds, 1996). Se calculó el índice de masa corporal (IMC, peso corporal, $\mathrm{kg}$ /estatura, $\mathrm{m}^{2}$ ) y la presión sanguínea media (PSM, presión sanguínea diastólica $+1 / 3$, presión sanguínea sistólica-presión sanguínea diastólica). El peso se registró con una báscula digital (Seca 813, Hamburgo, Alemania), la estatura con un estadímetro (Seca 206, Hamburgo, Alemania), la circunferencia de cintura (CC) con una cinta métrica flexible (Seca 201, Hamburgo, Alemania). El porcentaje de grasa corporal se calculó por impedancia bioeléctrica tetrapolar (Quantum X, Michigan USA) (Benton et al., 2011) posterior a 5 min de que el sujeto estuviera en una posición supina y con piernas y brazos separados sin tocarse el cuerpo, quitar todos los implementos de metal y limpiar la zona de los electrodos con etanol mientras se encuentra en esta posición. Los electrodos se pusieron sobre el lado derecho del dorso de la mano y pie. Todo lo anterior como lo explican procedimientos técnicos ya publicados (Benton et al., 2011; Lukaski et al., 1985). Antes de las mediciones el equipo se calibró con una resistencia de $500 \Omega$, la cual no varió más de $2 \Omega$. Las mediciones y entrevistas las aplicó un investigador calificado en ciencias de la salud y actividad física.

\section{3. Análisis estadísticos}

Todos los parámetros fueron expresados como medias \pm desviación estándar (DE) o porcentajes. Las diferencias entre grupos se analizaron por chi cuadrada, $t$ de medidas independientes, ANOVA de un factor y ANOVA multivariante, incluyéndose en este último la edad y el género como covariables. Las correlaciones se midieron por la correlación de Pearson. Para estudiar la importancia clínica de las diferencias estadísticas se calculó el tamaño del efecto para cada una de las variables. El tamaño del efecto para las diferencias entre grupos se analizó por la eta cuadrada $\left(\eta^{2}\right)$ y $f$ de Cohen; los valores $f$ alrededor de 0.10 se consideraron bajos, alrededor de 0.25 moderados y $\geq 0.40$ grandes (Ledesma et al., 2008). La validez interna del cuestionario de hábitos alimentarios se examinó por medio del alfa de Cronbach. Los análisis se efectuaron con el programa estadístico SPSS ver 22.0 y por el software libre $\mathrm{G}^{*}$ Power versión 3.1,[1] este último para calcular el tamaño de los efectos.

\subsection{Resultados}

Los sujetos sedentarios presentaron mayor peso corporal, estatura y CC; en cambio, registraron menor presión sanguínea sistólica (cuadro 1). Los participantes moderadamente activos presentaron mayor porcentaje de grasa corporal y reportaron realizar en promedio $1137 \pm 220 \mathrm{MET}-\mathrm{min} /$ semana de actividades físicas deportivas o recreativas, y los muy activos $2173 \pm 1101 \mathrm{MET}$-min/semana (cuadro 1). Aunque bajas las correlaciones, el nivel de actividad física realizado fue la variable que mejor identificó la presencia de enfermedades autorreportadas $(r=-0.36, p<$ $0.01)$, incluso por encima del IMC $(r=0.26, p<0.01)$ y CC $(r=0.23, p<0.01$, cuadro 5$)$. La edad y el sexo no se correlacionaron de manera significativa con ninguna de las variables del estado de salud autorreportadas (datos no mostrados). Sin embargo, entre las variables medidas a mayor edad menor IMC $(r=-0.16, p<0.05)$, mayor 
presión sanguínea sistólica $(r=0.28, p<0.01)$ y mayor presión sanguínea media $(r=0.23 p<0.01)$. Por otro lado, los hombres presentaron mayores valores de CC ( $99 \pm 12$ y $92 \pm 9 \mathrm{~cm}, p<0.01$, para hombres y mujeres respectivamente) y menor porcentaje de grasa corpzoral (29 $\pm 5 \%$ y $36 \pm 6 \%, p<0.01$, para hombres y mujeres respectivamente). Cabe señalar que el porcentaje de grasa corporal sólo fue predictivo de problemas de vesícula $(r=0.16, p<0.05)$ y artritis $(r=-0.17, p<0.05)$.

De acuerdo con el tamaño del efecto, las características físicas de los sujetos fueron diferentes entre grupos, ya que si bien las magnitudes de dichas diferencias fueron pequeñas ( $3 \%$ al $7 \%$; valor de $\eta^{2}$ ), el valor $f$ fue mayor de 0.25 (cuadro 1). El grupo de sedentarios reportó mayor número de padecimientos como falta de energía, estrés, gastritis, hipercolesterolemia y problemas circulatorios (cuadro 1 y cuadro $2 ; f=0.50$ ).

Los participantes sedentarios mencionaron que comen mayor cantidad de carnes rojas y blancas, huevos, aceite de oliva, sodas, jugos comerciales, café, crema, azúcar, burritos, lonches y galletas de harina refinada $(f>$ 0.25; cuadro 3). La sensibilidad y especificidad de los METs para determinar problemas de salud fue entre bajo y moderado, que es el punto de corte para la suma de enfermedades de 1137 METs (cuadro 4).

Por último, el cuestionario de 19 reactivos de hábitos alimentarios mostró una apropiada validez interna (alfa de Cronbach de 0.84).

\subsection{Discusión}

Las claves para conservar la salud y prolongar la vida son mantenerse físicamente activo y llevar una alimentación sana (Artinian et al., 2010; Haskell et al., 2007). Sin embargo, las circunstancias socioeconómicas, culturales, desconocimiento de hábitos saludables, falta de espacios para la práctica deportiva y hacinamiento en las grandes ciudades impiden muchas veces mantener un estilo de vida saludable ocasionando diversas enfermedades que afectan la calidad de vida (Marmot, 2005; Thow et al., 2010). Son tantos y diversos los problemas que acarrea el sedentarismo que incluso se ha propuesto crear un campo de estudio llamado fisiología del sedentarismo (Tremblay et al., 2010). Aquí se estudian estos dos principios saludables (actividad física y alimentación saludable) y cómo se relacionan con la salud en una muestra de adultos $\geq 50$ años, sedentarios y físicamente activos.

Se observa que, de acuerdo con el grupo etario, en promedio todos los participantes se encontraban en normopeso (IMC 22.4-31 kg/m², CC 88-103 cm) (Bulla, 2006; Woo et al., 2002). Además, aunque pequeñas las diferencias $\left(\eta^{2}\right.$ promedio $\left.=0.045\right)$, los sujetos sedentarios presentaron mayor IMC, CC y menor presión sanguínea sistólica $(f>0.25)$. Las personas moderadamente activas o muy activas reportaron 2.5 veces menor número de enfermedades que las personas sedentarias. Esto último significa que para tener buena salud en esta población no importa la cantidad de actividad física realizada, sino mantenerse físicamente activo; esta situación lo demuestra tanto el punto de corte en METs de actividad física (1 $137 \mathrm{MET} / \mathrm{min} / \mathrm{semana}$, personas moderadamente activas) como también las mayores correlaciones entre el estado de salud autorreportado y el nivel de actividad física realizada ( $r$ promedio $=-0.36$ ). Se ha demostrado que a mayor cantidad de ejercicio físico, mayores los beneficios en la salud (Hamilton et al., 2008; Haskell et al., 2007; Tremblay et al., 2010); en nuestro caso esta asociación, aunque significativa, sigue siendo baja $(r<0.4)$. Una razón de la baja asociación entre la autopercepción del estado de salud y la cantidad de actividad física realizada es que independientemente del tipo o nivel de ejercicio físico realizado las personas deportistas se perciben saludables y procuran mantener mejores hábitos saludables (Cobb-Clark et al., 2014), por ejemplo mejor calidad nutricional como aquí se observa (menor cantidad de alimentos procesados y con alto contenido de azúcares) (Jiménez et al., 2008).

Los adultos mayores suelen ser los más susceptibles a las inequidades y a los efectos de estilos de vida poco saludables (González-González et al., 2011). En especial, en el norte de México se observan altos índices de obesidad (37.8\%) (INSP, 2016) y hábitos alimenticios no recomendados, ya que ingieren una gran cantidad de alimentos procesados y con alto contenido de azúcares; de aquí la importancia de este estudio que muestra cómo afecta el estilo de vida a la salud de los adultos $\geq 50$ años. 
Por otro lado, también se observa que las personas físicamente activas comieron menos veces por semana tanto alimentos recomendables (carnes rojas y blancas, huevos, aceite de oliva) como no recomendables (refrescos, jugos comerciales, café, crema, azúcar, burritos y galletas de harina refinada) $(f>0.25)$ favoreciendo tener ligeramente menor IMC $(f=0.28)$ y menor CC $(f=0.38)$. Las razones de la menor cantidad de alimentos ingeridos en las personas físicamente activas no las sabemos, pero las porciones de dichos alimentos en esta población (no estudiadas) para satisfacer sus necesidades calóricas pudieran ser mayores a la de los sedentarios. De acuerdo con la ENSANUT MC 2016, la población adulta del norte de México consume mayor diversidad de alimentos recomendables ( 4.4 de 7 ) que no recomendables ( 2.3 de 6 ) respecto al resto del país (4.2 grupos de alimentos recomendables vs. 2.3 de no recomendables). En ese estudio, los alimentos fueron clasificados por sus características nutrimentales y a la evidencia de que podrían asociarse positiva o negativamente con el sobrepeso y la obesidad. La mayor preferencia de alimentos saludables sugiere una buena conciencia en la población sobre las causas y consecuencias del consumo inadecuado y exagerado de alimentos, ya que la ingesta de alimentos no recomendables está asociada con mayor prevalencia de sobrepeso y obesidad (Gutiérrez-Fisac et al., 2006).

En relación con el consumo de cereales, verduras y frutas no se observaron diferencias significativas entre los grupos evaluados; sin embargo, su consumo sigue estando por debajo de las recomendaciones nutricionales establecidas en el plato del buen comer (DOF, 2013). En un estudio realizado en 2007 en estudiantes universitarios del norte de México se encontró que sólo 13.2\% consume frutas diariamente y 15.6\% verduras. Los autores de este estudio argumentaron que este mal hábito alimenticio pudiera denotar la falta de acceso a estos alimentos, pues se prefieren en ocasiones otros de fácil preparación y fácil acceso (Rodriguez Tadeo y Rene, 2007). Las principales barreras reportadas en México para el consumo de alimentos saludables son la falta de dinero para comprar frutas y verduras ( $50.4 \%$ de los encuestados), la falta de conocimiento para preparar alimentos saludables (38.4\%), la falta de tiempo para preparar o consumir esta clase de alimentos (34.4\%), la falta de una alimentación saludable en la familia (32.4\%), la preferencia por consumir bebidas azucaradas y comida densa en energía (pastelillos, dulces y botanas, como papitas y frituras) (31.6\%), la falta de motivación para consumir alimentos saludables (28.3\%) y el desagrado por el sabor de las verduras (23\%) (INSP, 2016). De acuerdo con la ENSANUT MC 2016, casi la totalidad de la población está de acuerdo en que la obesidad está relacionada con factores de comportamiento como tomar muchas bebidas azucaradas (88.3\%), no comer suficientes verduras y frutas (84.3\%), pasar mucho tiempo en actividades sedentarias como ver la televisión o usar la computadora (89.3\%) y no realizar suficiente actividad física (94.8\%) (INSP, 2016). En nuestro, caso la cuestión educacional no fue un factor relacionado con los incorrectos hábitos alimentarios, ya que todos los participantes eran profesionistas.

Las posibles debilidades del estudio son las siguientes: a) utilizar un cuestionario de hábitos alimentarios pendiente de validar; sin embargo, aquí se observa una buena consistencia interna del mismo (alfa de Cronbach $\geq 0.80$ ). Otros cuestionarios similares han encontrado alfas de Cronbach entre 0.71-0.97 para sus diferentes dimensiones (Guyonnet et al., 2008; Sharma et al., 2014).b) Falta de datos socioeconómicos de la población; no obstante, como ya se mencionó, toda la población estudiada eran profesionistas trabajando actualmente o jubilados. $c$ ) Escasa muestra que fortaleciera los hallazgos. $d$ ) Falta de estudios clínicos que validaran el estado de salud de los participantes. e) Falta de mediciones de la ingesta calórica de los participantes.

Por último, es común que en la mayoría de reportes para detectar diferencias (significancia de la hipótesis nula) y asociaciones entre tratamientos y poblaciones apliquen estadística convencional para saber el valor de $p$ sin interesarse si las diferencias encontradas son de suficiente magnitud (precisión de la magnitud estimada) para considerarse importantes; se sugiere por ello que además de encontrar el valor de $p$, conocer también la magnitud de tales diferencias, o sea el tamaño de los efectos. Este estadístico también es utilizado si los datos analizados no presentan una distribución normal, el tamaño de la muestra entre tratamientos es diferente y los datos no son independientes, entre otras suposiciones (Lakens, 2013; Nakagawa y Cuthill, 2007). En este trabajo ocurren algunos de estos problemas, por lo que, además de la estadística clásica, analizamos el tamaño de los efectos. 


\section{Conclusiones}

La práctica de actividad física deportiva o recreativa, aunado al consumo de alimentos saludables, está asociada a un mejor estado de salud, menor número de enfermedades e impedimentos físicos en adultos $\geq 50$ años; además, a mayor edad menor IMC, pero mayor presión sanguínea. Las mujeres presentan menor CC, pero mayor porcentaje de grasa corporal. Sugerimos incrementar políticas en servicios de salud para establecer un ambiente cultural, física y nutricionalmente saludable y, de esta manera, contribuir a la disminución de enfermedades crónicas no transmisibles en la edad adulta.

\section{ANÁlisis Prospectivo}

Las crecientes presiones socioeconómicas favorecen estilos de vida no saludables, sedentarismo, incorrectos hábitos alimentarios, obesidad y diversas patologías que aumentan las disparidades y las discapacidades en la edad adulta (Sparling et al., 2015; Sun et al., 2013; Smith et al., 2010).

Dado el creciente aumento de la edad poblacional nuestros resultados, junto con los de la literatura ya expuesta, muestran que los gobiernos deben ser más responsables en sus políticas económicas y de salud, en crear campañas de información alimentaria más eficientes y en facilitar a la población espacios suficientes para la práctica sistemática del ejercicio físico. Sin embargo, la clase política mexicana no se encuentra aún convencida de los beneficios del deporte, ya que al menos a nivel educativo básico sigue siendo una hora semanal la duración de la clase de educación física y dos horas en secundaria (Diario Oficial de la Federación, 2018), por lo cual proponemos estudios nacionales que aborden de manera multidisciplinaria el estudio de los estilos de vida, hábitos alimentarios y actividad física en nuestra población para tener elementos convincentes que favorezcan políticas saludables.

\section{REFERENCIAS}

ACSM (American College of Sports Medicine). (2013). ACSM's guidelines for exercise testing and prescription. Lippincott Williams \& Wilkins.

Artinian, N. T., Fletcher, G. F., Mozaffarian, D., Kris-Etherton, P., Van Horn, L., Lichtenstein, A. H., (...) Redeker, N. S. (2010). Interventions to promote physical activity and dietary lifestyle changes for cardiovascular risk factor reduction in adults. A scientific statement from the American Heart Association. Circulation, 122, 406-441.

Barquera, S., Rivera-Dommarco, J. y Gasca-García, A. (2001). Políticas y programas de alimentación y nutrición en México. Salud Pública de México, 43(5), 464-477.

Benton, M. J., Swan, P. D., Schlairet, M. C. \& Sanderson, S. (2011). Comparison of body composition measurement with whole body multifrequency bioelectrical impedance and air displacement plethysmography in healthy middle-aged women. Health Care for Women International, 32(12), 1068-1078.

Bimbo, F., Bonanno, A. \& Viscecchia, R. (2015). Adult obesity, food access, and eating habits in Italy: An empirical analysis. British Food Journal, 117(6), 1689-1705.

Bulla, F. B. (2006). Tendencias actuales en la valoración antropométrica del anciano. Revista de la Facultad de Medicina, 54(4), 283-289.

Cobb-Clark, D. A., Kassenboehmer, S. C. \& Schurer, S. (2014). Healthy habits: The connection between diet, exercise, and locus of control. Journal of Economic Behavior \& Organization, 98, 1-28. 
Craig, C. L., Marshall, A. L., Sjöström, M., Bauman, A. E., Booth, M. L., Ainsworth, B. E., (...) Sallis, J. F. (2003). International physical activity questionnaire: 12-country reliability and validity. Medicine \& Science in Sports \& Exercise, 35(8), 1381-1395.

DOF (Diario Oficial de la Federación). (2018). Acuerdo número 10/05/18 por el que se emiten los lineamientos de ajuste a las horas lectivas señaladas en el diverso número 592 por el que se establece la articulación de la educación básica para el ciclo escolar 2018-2019. Disponible en http://www.dof.gob.mx/nota_detalle. php? codigo $=5525412 \&$ fecha $=07 / 06 / 2018$.

DOF (Diario Oficial de la Federación). (2013). Norma Oficial Mexicana NOM-043-SSA2-2012, Servicios básicos de salud. Promoción y educación para la salud en materia alimentaria. Criterios para brindar orientación. Disponible en http://dof.gob.mx/nota_detalle.php?codigo=5285372\&fecha=22/01/2013

Drewnowski, A., \& Evans, W. J. (2001). Nutrition, physical activity, and quality of life in older adults summary. The Journals of Gerontology Series A: Biological Sciences and Medical Sciences, 56(suppl 2), 89-94.

Ford, E. S., Kohl, H. W., Mokdad, A. H., \& Ajani, U. A. (2005). Sedentary behavior, physical activity, and the metabolic syndrome among US adults. Obesity, 13(3), 608-614.

GBD (Global Burden Disease) 2015 Risk Factors Collaborators. (2016). Global, regional, and national comparative risk assessment of 79 behavioural, environmental and occupational, and metabolic risks or clusters of risks in 188 countries, 1990-2013: A systematic analysis for the Global Burden of Disease Study 2013. The Lancet, 386(10010), 2287-2323. Disponible en https://www.sciencedirect.com/ science/article/pii/S0140673616316798

González-González, C., Sánchez-García, S., Juárez-Cedillo, T., Rosas-Carrasco, O., Gutiérrez-Robledo, L. M., \& García-Peña, C. (2011). Health care utilization in the elderly Mexican population: Expenditures and determinants. BMC Public Health, 11(1), 192.

Goodpaster, B. H., DeLany, J. P., Otto, A. D., Kuller, L., Vockley, J., South-Paul, J. E., (...) Hames, K. C. (2010). Effects of diet and physical activity interventions on weight loss and cardiometabolic risk factors in severely obese adults: A randomized trial. JAMA, 304(16), 1795-1802.

Gutierres, J. P., Rivera-Dommarco, J., Shamah-Levy, T., Villalpando-Hernández, S., Franco, A., Cuevas-Nasu, L., (...) Hernández-Ávila, M. (2012). Encuesta Nacional de Saludy Nutrición 2012: Resultados Nacionales. Cuernavaca México: Institutio Nacional de Salud Pública. Disponible en http://ensanut.insp.mx/ informes/ENSANUT2012ResultadosNacionales.pdf.

Gutiérrez-Fisac, J. L., Royo-Bordonada, M. Á. y Rodríguez-Artalejo, F. (2006). Riesgos asociados a la dieta occidental y al sedentarismo: la epidemia de obesidad. Gaceta sanitaria, 20, 48-54.

Guyonnet, D., Chassany, O., Picard, C., Guillemin, I., Meunier, J., Seignobos, E., (...) Trudeau, E. (2008). Perceived subject outcomes and impact on health-related quality of life associated with diet using the new Food Benefits Assessment (FBA@) questionnaire: Development and psychometric validation. Public Health Nutrition, 11(11), 1163-1172.

Hamilton, M. T., Healy, G. N., Dunstan, D. W., Zderic, T. W., \& Owen, N. (2008). Too little exercise and too much sitting: Inactivity physiology and the need for new recommendations on sedentary behavior. Current Cardiovascular Risk Reports, 2(4), 292-298.

Haskell, W. L., Lee, I.-M., Pate, R. R., Powell, K. E., Blair, S. N., Franklin, B. A., (...) Bauman, A. (2007). Physical activity and public health. Updated recommendation for adults from the American College of Sports Medicine and the American Heart Association. Circulation, 116, 1081-1093.

Hoffman, D. J. (2010). Early nutrition and adult health: Perspectives for international and community nutrition programs and policies. Nutrition Research and Practice, 4(6), 449-454. 
INEGI (Instituto Nacional de Estadística Geografía e Informática). (2013). Censo de población y vivienda 2010. Perfil sociodemográfico: Estados Unidos Méxicanos. México: Instituto Nacional de Estadística y Geografía. Disponible en http://internet.contenidos.inegi.org.mx/contenidos/Productos/prod_serv/contenidos/ espanol/bvinegi/productos/censos/poblacion/2010/perfil_socio/uem/702825047610_1.pdf.

INSP (Instituto Nacional de Salud Pública). (2016). Encuesta Nacional de Salud de Nutrición de Medio Camino 2016: Informe final de resultados. México: Secretaría de Salud. Disponible en http://www.gob.mx/cms/ uploads/attachment/file/209093/ENSANUT.pdf.

Jiménez, M. G., Martínez, P., Miró, E. y Sánchez, A. I. (2008). Bienestar psicológico y hábitos saludables: ¿están asociados a la práctica de ejercicio físico? International Journal of Clinical and Health Psychology, $8(1), 185-202$.

Kushner, R. F., \& Choi, S. W. (2010). Prevalence of unhealthy lifestyle patterns among overweight and obese adults. Obesity, 18(6), 1160-1167.

Lakens, D. (2013). Calculating and reporting effect sizes to facilitate cumulative science: A practical primer for t-tests and ANOVAs. Frontiers in Psychology, 4(863), 1-12. http://10.3389/fpsyg.2013.00863

Ledesma, R., Macbeth, G. y Cortada de Kohan, N. (2008). Tamaño del efecto: revisión teórica y aplicaciones con el sistema estadístico ViSta. Revista Latinoamericana de Psicología, 40(3), 425-439.

Lukaski, H. C., Johnson, P. E., Bolonchuk, W. W., \& Lykken, G. I. (1985). Assessment of fat-free mass using bioelectrical impedance measurements of the human body. The American Journal of Clinical Nutrition, 41(4), 810-817.

Marmot, M. (2005). Social determinants of health inequalities. The Lancet, 365(9464), 1099-1104.

Medina, C., Janssen, I., Campos, I., \& Barquera, S. (2013). Physical inactivity prevalence and trends among Mexican adults: Results from the National Health and Nutrition Survey (ENSANUT) 2006 and 2012. BMC Public Health, 13(1), 1063.

Nakagawa, S., \& Cuthill, I. C. (2007). Effect size, confidence interval and statistical significance: A practical guide for biologists. Biological Reviews, 82(4), 591-605.

Nelson, M. E., Rejeski, W. J., Blair, S. N., Duncan, P. W., Judge, J. O., King, A. C., (...) Castaneda-Sceppa, C. (2007). Physical activity and public health in older adults: Recommendation from the American College of Sports Medicine and the American Heart Association. Circulation, 116, 1094-1105.

Norton, K., y Olds, T. (1996). Anthropometrica: A textbook of body measurement for sports and health courses. UNSW press.

Owen, N., Healy, G. N., Matthews, C. E., \& Dunstan, D. W. (2010). Too much sitting: The population-health science of sedentary behavior. Exercise and Sport Sciences Reviews, 38(3), 105-113.

Pino, J. L., Díaz, C. y López, M. Á. (2011). Construcción y validación de un cuestionario para medir conductas y hábitos alimentarios en usuarios de la atención primaria de salud. Revista Chilena de Nutrición, $38(1), 41-51$.

Rodriguez Tadeo, A. y Rene, U. (2007). Hábitos alimenticios poco saludables en estudiantes Universitarios Fronterizos. Avances, 147, 1-13.

Sharma, S., Roberts, L., \& Fleming, S. (2014). Nutrition self-efficacy assessment: Development of a questionnaire and evaluation of reliability in African-American and Latino children.Journal of Human Nutrition and Dietetics, 27(s2), 160-166.

Smith, R., Rabadán-Diehl, C, Cravioto, A., Wall-Medrano, A., Chapman, S., De Pinho, K., Enkin, M., Gillies, J., Guerra, J. A., Gupta, R., Gusi, N., Lijing, Y., Marcet, B., Miranda, J. J., Rubinstein, A., Sevick, M. A., 
Upshur, R. (2010). Prevention and health promotion. In: A. R. Jadad, A. Cabrera, F. Martos, R. Smith, R. F. Lyons When people live with multiple chronic diseases: A collaborative approach to an emerging global challenge (pp. 57-85). Granada (Spain): Andalusian School of Public Health. Disponible en https://www.opimec.org/media/files/BOOK_OPIMEC_100818.pdf

Sparling, P. B., Howard, B. J., Dunstan, D. W., \& Owen, N. (2015). Recommendations for physical activity in older adults. BMJ: British Medical Journal (Online), 350.

Sun, F., Norman, I. J., \& While, A. E. (2013). Physical activity in older people: A systematic review. BMC Public Health, 13(1), 449.

Thow, A. M., Jan, S., Leeder, S., \& Swinburn, B. (2010). The effect of fiscal policy on diet, obesity and chronic disease: A systematic review. Bulletin of the World Health Organization, 88(8), 609-614.

Tourlouki, E., Matalas, A.-L., \& Panagiotakos, D. B. (2009). Dietary habits and cardiovascular disease risk in middle-aged and elderly populations: A review of evidence. Clinical interventions in Aging, 4, 319

Tremblay, M. S., Colley, R. C., Saunders, T. J., Healy, G. N., \& Owen, N. (2010). Physiological and health implications of a sedentary lifestyle. Applied Physiology, Nutrition, and Metabolism, 35(6), 725-740.

Varo, J. J., Martínez-González, M. A., De Irala-Estévez, J., Kearney, J., Gibney, M., \& Martínez, J. A. (2003). Distribution and determinants of sedentary lifestyles in the European Union. International Journal of Epidemiology, 32(1), 138-146.

Woo, J., Ho, S. C., Yu, A. L. M., \& Sham, A. (2002). Is waist circumference a useful measure in predicting health outcomes in the elderly? International Journal of Obesity, 26(10), 1349-1355.

\section{Anexo}

CUADRO 1

Características físicas de los sujetos

\begin{tabular}{lccccc}
\hline & Sedentarios & $\begin{array}{c}\text { Moderadamente } \\
\text { activos }\end{array}$ & Muy activos & \multicolumn{2}{c}{ Tamaño del efecto } \\
& & & $\eta^{2}$ & $f$ \\
\hline Edad (años) & $57.7 \pm 5.5$ & $60.0 \pm 7.8$ & $57.4 \pm 7.5$ & 0.029 & 0.28 \\
Estatura (m) & $1.70 \pm 0.12$ & $1.64 \pm 0.10^{\mathrm{a}}$ & $1.68 \pm 0.10$ & 0.048 & 0.33 \\
Peso (kg) & $86.1 \pm 23.4$ & $76.5 \pm 12.4^{\mathrm{a}}$ & $78.0 \pm 12.1^{\mathrm{a}}$ & 0.059 & 0.35 \\
IMC (kg/m²) & $29.6 \pm 5.5$ & $28.4 \pm 3.6$ & $27.8 \pm 3.2^{\mathrm{a}}$ & 0.028 & 0.28 \\
CC (cm) & $100.6 \pm 14.4$ & $93.8 \pm 9.3^{\mathrm{a}}$ & $93.8 \pm 8.5^{\mathrm{a}}$ & 0.072 & 0.38 \\
Grasa corporal (\%) & $32.0 \pm 6.5$ & $34.7 \pm 6.4^{\mathrm{a}}$ & $30.9 \pm 5.8^{\mathrm{b}}$ & 0.069 & 0.38 \\
PSS (mmHg) & $123 \pm 12$ & $129 \pm 14^{\mathrm{a}}$ & $128 \pm 13$ & 0.034 & 0.29 \\
PSD (mmHg) & $84 \pm 11$ & $83 \pm 11$ & $88 \pm 17$ & 0.027 & 0.27 \\
PSM (mmHg) & $104 \pm 11$ & $106 \pm 10$ & $108 \pm 13$ & 0.024 & 0.26 \\
MET-min/semana & 0 & $1137 \pm 220$ & $2173 \pm 1101$ & 0.618 & 0.50 \\
Número de enfermedades & $4.3 \pm 2.4$ & $1.7 \pm 2.1^{\mathrm{a}}$ & $1.8 \pm 2.1^{\mathrm{a}}$ & 0.204 & 0.50 \\
\hline
\end{tabular}

Nota: prueba ANOVA multivariante. Los valores se presentan como medias \pm DE. CC $=$ circunferencia de cintura, IMC $=$ índice de masa corporal, $\mathrm{PSS}=$ presión sanguínea sistólica, $\mathrm{PSD}=$ presión sanguínea diastólica, $\mathrm{PSM}=$ presión sanguínea media, MET = equivalente metabólico (por sus siglas en inglés). ${ }^{*} p<0.05 . \mathrm{a}=$ diferente de sedentarios, $\mathrm{b}=$ diferente de activos.

Fuente: elaboración propia a partir de los resultados. 
CUADRO 2

Padecimientos e impedimentos físicos que presentaron los participantes

\begin{tabular}{|lccccc}
\hline & Sedentarios & $\begin{array}{c}\text { Moderadamente } \\
\text { activos }\end{array}$ & Muy activos & Chi cuadrada & $\begin{array}{c}\text { Tamaño del efecto } \\
\eta^{2}\end{array}$ \\
\hline Falta de energía & $42 \%$ & $12 \%$ & $7.5 \%$ & 0.000 & 0.132 \\
Estrés & $56 \%$ & $17 \%$ & $25 \%$ & 0.000 & 0.119 \\
Ansiedad & $20 \%$ & $7 \%$ & $4 \%$ & 0.012 & 0.045 \\
Gastritis & $22 \%$ & $5 \%$ & $6 \%$ & 0.002 & 0.061 \\
Úlcera & $2 \%$ & $1 \%$ & $0 \%$ & 0.544 & 0.006 \\
Alergias & $20 \%$ & $16 \%$ & $12 \%$ & 0.491 & 0.007 \\
Colesterol alto & $34 \%$ & $13 \%$ & $10 \%$ & 0.002 & 0.064 \\
Anemia & $10 \%$ & $2 \%$ & $0 \%$ & 0.011 & 0.045 \\
Problemas de vesícula & $0 \%$ & $2 \%$ & $3 \%$ & 0.491 & 0.007 \\
Problemas de riñón & $10 \%$ & $4 \%$ & $8 \%$ & 0.325 & 0.011 \\
Presión alta & $38 \%$ & $22 \%$ & $18 \%$ & 0.032 & 0.034 \\
Problemas de corazón & $6 \%$ & $7 \%$ & $3 \%$ & 0.518 & 0.007 \\
Diabetes & $18 \%$ & $7 \%$ & $6 \%$ & 0.059 & 0.028 \\
Cáncer & $2 \%$ & $1 \%$ & $1 \%$ & 0.935 & 0.000 \\
Retención de líquidos & $14 \%$ & $8 \%$ & $8 \%$ & 0.447 & 0.008 \\
Mala circulación & $32 \%$ & $6 \%$ & $10 \%$ & 0.000 & 0.092 \\
Calambres & $28 \%$ & $5 \%$ & $16 \%$ & 0.001 & 0.070 \\
Diarrea & $4 \%$ & $4 \%$ & $3 \%$ & 0.955 & 0.000 \\
Estreñimiento & $14 \%$ & $6 \%$ & $6 \%$ & 0.196 & 0.016 \\
Dolor de cabeza & $16 \%$ & $4 \%$ & $7 \%$ & 0.038 & 0.033 \\
Artritis & $12 \%$ & $5 \%$ & $7 \%$ & 0.314 & 0.012 \\
Dolores óseos & $12 \%$ & $4 \%$ & $13 \%$ & 0.078 & 0.026 \\
Celulitis & $18 \%$ & $11 \%$ & $6 \%$ & 0.120 & 0.021 \\
\hline
\end{tabular}

Fuente: elaboración propia a partir de los resultados.

CUADRO 3

Hábitos alimentarios de los participantes por nivel de actividad física

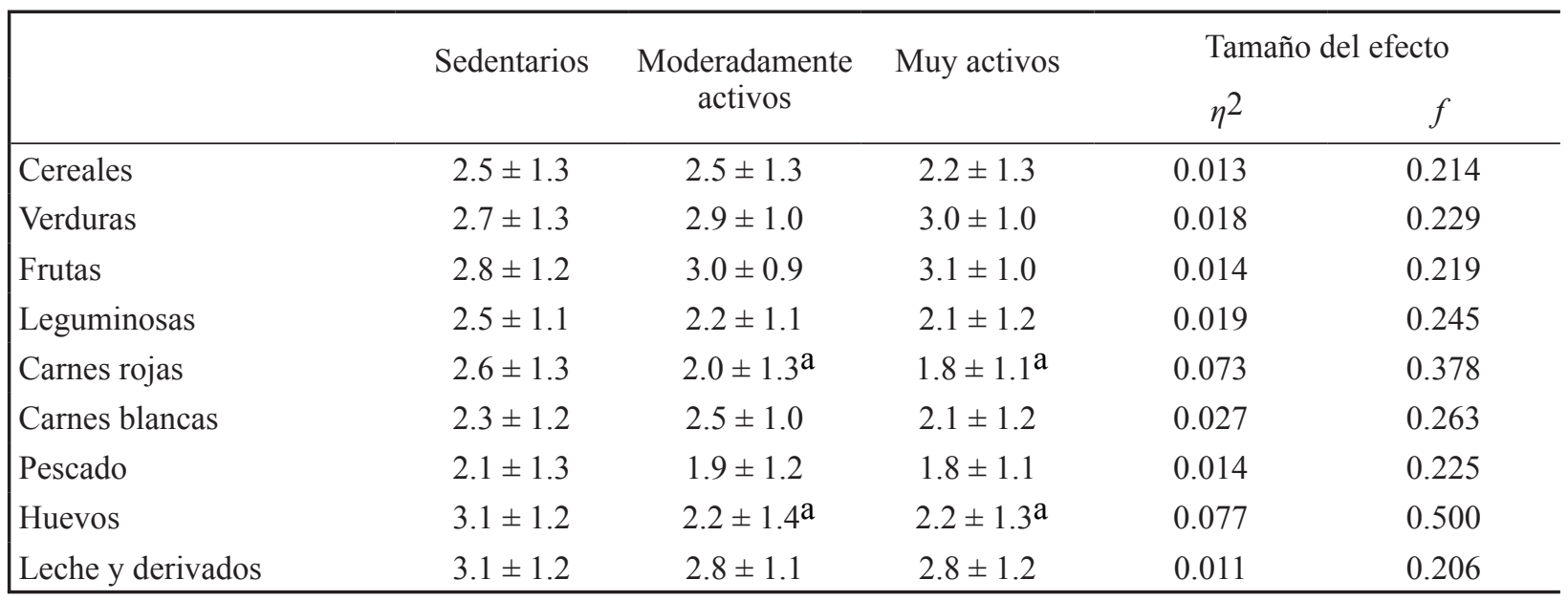


CUADRO 3 (continúa)

Hábitos alimentarios de los participantes por nivel de actividad física

\begin{tabular}{|c|c|c|c|c|c|}
\hline Aceite de oliva & $2.5 \pm 1.6$ & $2.3 \pm 1.6$ & $1.8 \pm 1.6^{\mathrm{a}}$ & 0.027 & 0.272 \\
\hline Sodas & $2.0 \pm 1.6$ & $1.1 \pm 1.4^{\mathrm{a}}$ & $1.1 \pm 1.2^{\mathrm{a}}$ & 0.072 & 0.378 \\
\hline Jugos comerciales & $1.8 \pm 1.6$ & $1.3 \pm 1.5$ & $1.1 \pm 1.3^{\mathrm{a}}$ & 0.028 & 0.271 \\
\hline Café & $2.3 \pm 1.6$ & $1.6 \pm 1.3^{\mathrm{a}}$ & $1.1 \pm 1.1^{\mathrm{a}, \mathrm{b}}$ & 0.107 & 0.500 \\
\hline Crema & $2.0 \pm 1.6$ & $1.3 \pm 1.2$ & $1.0 \pm 1.1^{\mathrm{a}}$ & 0.090 & 0.500 \\
\hline Azúcar & $2.2 \pm 1.6$ & $1.6 \pm 1.3$ & $1.0 \pm 1.1^{\mathrm{a}}$ & 0.101 & 0.500 \\
\hline Burritos y lonches & $1.7 \pm 1.4$ & $1.1 \pm 1.4$ & $0.9 \pm 1.0^{\mathrm{a}}$ & 0.047 & 0.380 \\
\hline Dulces & $1.3 \pm 1.3$ & $1.0 \pm 1.2$ & $1.0 \pm 1.0$ & 0.011 & 0.198 \\
\hline Galletas integrales & $1.3 \pm 1.4$ & $1.3 \pm 1.2$ & $1.2 \pm 1.1$ & 0.003 & 0.108 \\
\hline Galletas de harina refinada & $1.9 \pm 1.6$ & $1.5 \pm 1.5$ & $1.2 \pm 1.3^{\mathrm{a}}$ & 0.041 & 0.311 \\
\hline
\end{tabular}

Nota: prueba ANOVA de un factor. Los valores representan veces por semana en ingerir el alimento: medias $\pm \mathrm{DE} .{ }^{*} p<0.05$.

a diferente vs. sedentarios, ${ }^{b}$ diferente vs. moderadamente activos.

Fuente: elaboración propia a partir de los resultados.

\section{CUADRO 4}

Sensibilidad y especificidad de la actividad física (METs) para presentar problemas de salud

\begin{tabular}{lrcccc}
\hline & METs a & Sensibilidad & Especificidad & ABC & IY \\
\hline Falta de energía & 1194 & 0.57 & 0.83 & 0.75 & 0.40 \\
Estrés & 819 & 0.83 & 0.48 & 0.63 & 0.31 \\
Ansiedad & 1038 & 0.61 & 0.79 & 0.70 & 0.40 \\
Gastritis & 819 & 0.77 & 0.58 & 0.65 & 0.35 \\
Úlcera & 939 & 0.58 & 1.00 & 0.77 & 0.58 \\
Alergias & 1194 & 0.54 & 0.71 & 0.60 & 0.25 \\
Colesterol alto & 1194 & 0.55 & 0.74 & 0.67 & 0.29 \\
Anemia & 1194 & 0.52 & 1.00 & 0.79 & 0.52 \\
Problemas de vesícula & 1815 & 0.19 & 1.00 & 0.39 & 0.19 \\
Problemas de riñón & 819 & 0.75 & 0.39 & 0.54 & 0.14 \\
Presión alta & 819 & 0.78 & 0.39 & 0.60 & 0.17 \\
Problemas de corazón & 1335 & 0.50 & 0.73 & 0.59 & 0.23 \\
Diabetes & 819 & 0.77 & 0.47 & 0.64 & 0.24 \\
Cáncer & 939 & 0.58 & 0.67 & 0.54 & 0.25 \\
Retención de líquidos & 1038 & 0.59 & 0.58 & 0.60 & 0.17 \\
Mala circulación & 819 & 0.79 & 0.57 & 0.66 & 0.36 \\
Calambres & 819 & 0.78 & 0.48 & 0.58 & 0.26 \\
Diarrea & 194 & 0.51 & 0.71 & 0.50 & 0.22 \\
Estreñimiento & 819 & 0.77 & 0.44 & 0.61 & 0.21 \\
Dolor de cabeza & 819 & 0.76 & 0.50 & 0.61 & 0.26 \\
Artritis & 819 & 0.75 & 0.40 & 0.54 & 0.15 \\
Dolores óseos & 819 & 0.76 & 0.33 & 0.50 & 0.09 \\
Celulitis & 939 & 0.62 & 0.77 & 0.66 & 0.39 \\
Suma de enfermedades & 1137 & 0.83 & 0.53 & 0.69 & 0.36 \\
\hline
\end{tabular}

Nota: ${ }^{\mathrm{a}}=$ punto de corte en METs, METs = equivalente metabólico (por sus siglas en inglés). ABC = área bajo la curva ROC. $\mathrm{IY}=$ índice Youden.

Fuente: elaboración propia a partir de los resultados. 
CUADRO 5

Matriz de correlación entre variables antropométricas, actividad física y enfermedades autorreportadas

\begin{tabular}{llclc}
\hline & $\mathrm{NAF}$ & $\mathrm{IMC}$ & $\mathrm{CC}$ & $\% \mathrm{GC}$ \\
\hline Falta de energía & $-0.33^{* *}$ & $0.21^{* *}$ & 0.13 & 0.11 \\
Estrés & $-0.23^{* *}$ & $0.19^{* *}$ & 0.09 & -0.01 \\
Ansiedad & $-0.19^{* *}$ & 0.10 & 0.12 & -0.01 \\
Gastritis & $-0.19^{* *}$ & -0.06 & 0.04 & -0.13 \\
Úlcera & -0.08 & 0.02 & 0.08 & -0.04 \\
Alergia & -0.08 & -0.02 & -0.02 & 0.06 \\
Colesterol alto & $-0.23^{* *}$ & $0.22^{* *}$ & $0.15^{*}$ & 0.11 \\
Anemia & $-0.20^{* *}$ & -0.01 & -0.01 & -0.10 \\
Problemas de vesícula & 0.08 & 0.04 & -0.04 & $0.16^{*}$ \\
Problemas de riñón & -0.03 & 0.12 & $0.15^{*}$ & -0.01 \\
Hipertensión arterial & $-0.17^{*}$ & $0.17^{*}$ & $0.22^{* *}$ & 0.07 \\
Problemas de corazón & -0.06 & 0.09 & 0.06 & -0.02 \\
Diabetes & $-0.15^{*}$ & 0.07 & 0.03 & -0.02 \\
Cáncer & -0.01 & $0.15^{*}$ & $0.23^{* *}$ & 0.09 \\
Retención de líquidos & -0.08 & $0.32^{* *}$ & $0.20^{* *}$ & 0.13 \\
Mala circulación & $-0.22^{* *}$ & $0.28^{* *}$ & $0.25^{* *}$ & 0.12 \\
Calambres & -0.10 & $0.17^{*}$ & $0.20^{* *}$ & -0.07 \\
Diarrea & -0.02 & 0.08 & -0.02 & 0.05 \\
Estreñimiento & -0.12 & 0.05 & 0.03 & -0.02 \\
Dolores de cabeza & -0.12 & -0.10 & -0.01 & -0.08 \\
Artritis & -0.06 & -0.02 & 0.06 & $-0.17^{*}$ \\
Dolores óseos & 0.003 & -0.12 & -0.14 & -0.03 \\
Celulitis & $-0.14^{*}$ & 0.04 & -0.04 & 0.04 \\
Suma de enfermedades & $-0.36^{* *}$ & $0.26^{* *}$ & $0.23^{* *}$ & \\
\hline & & 0.03 & \\
\hline
\end{tabular}

Nota: $\mathrm{CC}=$ Circunferencia de cintura, $\% \mathrm{GC}=$ Porcentaje de grasa corporal, NAF $=$ Nivel de actividad física,

$\mathrm{IMC}=$ Índice de masa corporal. ${ }^{*} p<0.05,{ }^{* *} p<0.01$

Fuente: elaboración propia a partir de los resultados.

\section{Nota}

[1] Disponible en http://www.psychologie.hhu.de/arbeitsgruppen/allgemeine-psychologie-und-arbeitspsychologie/gpower.html.

\section{BY-NC-ND}

